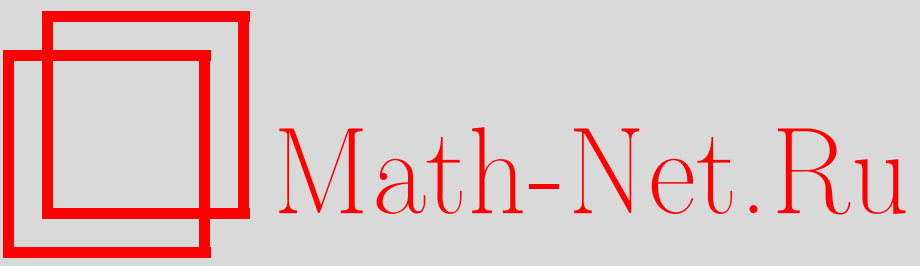

Б. Л. Воронов, И. В. Тютин, Ш. С. Шахвердиев, О локальных операторах в вариационных производных в теории поля, ТМФ, 1999, том 120, номер 2, 256-276

DOI: https://doi.org/10.4213/tmf774

Использование Общероссийского математического портала Math-Net.Ru подразумевает, что вы прочитали и согласны с пользовательским соглашением

http://www . mathnet.ru/rus/agreement

Параметры загрузки:

IP : 3.89 .185 .249

26 апреля 2023 г., 17:07:06 


\section{О ЛОКАЛЬНЫХ ОПЕРАТОРАХ В ВАРИАЦИОННЫХ ПРОИЗВОДНЫХ В ТЕОРИИ ПОЛЯ}

Развит математически последовательный формализм, в котором результат действия локальных дифференциальных операторов в вариационных производных высших порядков на локальные функционалы не содержит неопределенных выражений типа $\delta(0)$. Предлагаемый формализм применяется к формулировке Баталина-Вилковыского локальной калибровочной теории поля общего вида и ее $S p(2)$-симметричному обобщению. Обсуждается связь с квазиклассическим разложением.

\section{1. ВВЕДЕНИЕ}

В функциональном формализме локальной квантовой теории поля нередко приходится сталкиваться с трудностью, имеюшей жаргонное название "трудность $\delta(0)$ ". Суть ее может быть проиллюстрирована следуюшим простым примером. Пусть $S(\varphi)$ - локальньй функционал поля $\varphi(x)$, например действие,

$$
S(\varphi)=\int d x \mathcal{L}\left(\varphi(x), \partial_{\mu} \varphi(x), \ldots\right), \quad \partial_{\mu}=\frac{\partial}{\partial x^{\mu}} .
$$

Тогда вторая вариационная производная $\delta^{2} S(\varphi) / \delta \varphi\left(x_{1}\right) \delta \varphi\left(x_{2}\right)$ этого функционала есть квазилокальная обобщенная функция (уточнение понятия квазилокальной обобщенной функции см. в п. 2.3), символически $\delta^{2} S(\varphi) / \delta \varphi\left(x_{1}\right) \delta \varphi\left(x_{2}\right) \sim \delta\left(x_{1}-x_{2}\right)$. Соответственно локальные дифференциальные операторы в вариационных производных второго порядка вида

$$
\Delta_{2}=\int d x E_{2}\left(\varphi(x), \partial_{\mu} \varphi(x), \ldots\right) \frac{\delta}{\delta \varphi(x)} \frac{\delta}{\delta \varphi(x)}
$$

не определены на локальных функционалах: $\Delta_{2} S \sim \delta(0)$. Не определены такие операторы и на произведениях $S_{1}(\varphi) S_{2}(\varphi) \ldots$ локальных функционалов и вообще на функциях $f\left(S_{1}, S_{2}, \ldots\right)$ от последних, если принимать стандартные правила Лейбница

$$
\begin{aligned}
\Delta_{2}\left(S_{1}(\varphi) S_{2}(\varphi) \ldots\right)= & \left(\Delta_{2} S_{1}(\varphi)\right) S_{2}(\varphi) \ldots+ \\
& +2 \int d x E_{2}(\ldots) \frac{\delta S_{1}}{\delta \varphi(x)} \frac{\delta S_{2}}{\delta \varphi(x)} \ldots+S_{1}\left(\Delta_{2} S_{2}\right) \ldots+\cdots .
\end{aligned}
$$

* Физический институт им. П.Н. Лебедева РАН, Москва, Россия. E-mail: voronov@td.lpi.ac.ru; tyutin@td.lpi.ac.ru

${ }^{\dagger}$ Московский государственный университет, Москва, Россия. E-mail: shahv@td.lpi.ac.ru 
Аналогичные утверждения справедливы для высших вариационных производных $\delta^{n} S(\varphi) / \delta \varphi\left(x_{1}\right) \ldots \delta \varphi\left(x_{n}\right), n>2$, и соответственно для локальных дифференциальных операторов высшего порядка

$$
\Delta_{n}=\int d x E_{n}\left(\varphi(x), \partial_{\mu} \varphi(x), \ldots\right)\left(\frac{\delta}{\delta \varphi(x)}\right)^{n}
$$

Между тем появление подобных операторов неизбежно в локальной теории поля. Например, они возникают при рассмотрении локальных замен переменных в определяющих теорию функциональных интегралах, в частности при поисках инвариантной меры интегрирования в локальных калибровочных теориях общего вида. Более того, оператор вида $\Delta_{2}$ лежит в основании квантования Баталина-Вилковыского (БВ) калибровочных теорий [1]. Появляющаяся при этом величина $\delta(0)$ должна компенсироваться соответствующей “локальной мерой”, которая изначально сингулярна.

Случается, как например в теориях Янга-Миллса или суперсимметричных теориях, что коэффициент при $\delta(0)$ по специфическим алгебраическим причинам оказывается равным нулю с самого начала, тогда неопределенность $0 \times \infty$ разрешается в пользу нуля.

В обшем случае стандартньй способ обхода трудности $\delta(0)$ состоит в применении рассуждений типа: “при использовании размерной регуляризации соответствующая сингулярность, пропорциональная $\delta(0)$, равна нулю" [2]. Короче, принимается формальное правило " $\delta(0)=0$ " на промежуточных этапах формулировки. Успешный опыт работы с этим правилом заставляет предположить возможность такого математически последовательного формализма, в котором величина $\delta(0)$ не возникает вовсе. В данной работе мы реализуем такую возможность. В предлагаемом формализме избавление от $\delta(0)$ является следствием определений. Этот формализм применяется прежде всего к БВ-формулировке локальной калибровочной теории общего вида. Оказывается, что вся содержательная часть теории остается при этом неизменной.

Предлагаемый формализм специфичен именно для локальной теории поля и не имеет прямых аналогов в теории функций конечного числа переменных. Это лишний раз предостерегает [3] против возможных попыток оправдывать некоторые, иногда основополагаюшие, утверждения в локальной теории поля ссылками на конечномерные аналоги, тем более заменять доказательства такими ссылками. С другой стороны, как будет видно ниже, предлагаемый формализм реализует последовательное квазиклассическое (по постоянной Планка $\hbar$ ) квантование.

В разделе 2 дается определение рассматриваемых классов функционалов и операторов, формулируются правила действия операторов на функционалы и обсуждаются их свойства. В разделе 3 рассмотрены специальный класс операторов вида $\exp \nabla$ и специальный класс функционалов вида $\exp S$, а также связь предлагаемого формализма с квазиклассическим разложением. В разделе 4 рассмотрены локальные замены переменных. В разделе 5 обсуждается применение предлагаемого формализма к БВ-формулировке калибровочных теорий и в разделе 6 - к ее $S p(2)$-симметричному обобщению. 


\section{2. ФУНКЦИОНАЛЫ, ОПЕРАТОРЫ $\nabla$}

2.1. Поля. Исходные поля будем обозначать через $\Gamma=\left\{\Gamma^{A}(x)\right\}$ и использовать сокрашенные обозначения типа $\Gamma^{A_{k}}=\Gamma^{A_{k}}\left(x_{k}\right), E_{n}^{(A)_{n}}=E_{n}^{A_{1} \ldots A_{n}}\left(x_{1}, \ldots, x_{n} ; \Gamma\right)$. Поля принимают значения в алгебре Березина, включающей равноправно бозе- и ферми-поля, их грассмановы четности обозначаются через $\varepsilon\left(\Gamma^{A}\right)=\varepsilon_{A}$. Производные по полям левые,

$$
\frac{\delta}{\delta \Gamma^{A_{k}}\left(x_{k}\right)}=\delta_{A_{k}}, \quad \varepsilon\left(\delta_{A_{k}}\right)=\varepsilon_{A_{k}} .
$$

Кроме того, используются следуюшие сокрашения:

$$
\begin{gathered}
\left(\delta_{A}\right)^{n}=\delta_{A_{1}} \ldots \delta_{A_{n}}, \quad \varepsilon\left(\left(\delta_{A}\right)^{n}\right)=\sum_{k=1}^{n} \varepsilon_{A_{k}}, \\
(A)_{m}=A_{1} \ldots A_{m} .
\end{gathered}
$$

По повторяюшимся индексам подразумевается суммирование (интегрирование).

2.2. Функционалы. Сушественным для дальнейшего является ограничение на класс рассматриваемых функционалов $\Phi$ полей $Г$. Этот класс включает всевозможные гладкие локальные функционалы $S_{i}(\Gamma), i=1,2, \ldots$, с определенной грассмановой четностью $\varepsilon\left(S_{i}\right)=\varepsilon(i)$ вида

$$
S_{i}(\Gamma)=\int d x \mathcal{L}_{i}\left(x, \Gamma(x), \partial_{\mu} \Gamma(x), \ldots\right)
$$

где локальная плотность $\mathcal{L}_{i}$ - гладкая функция координат $x$, полей $\Gamma(x)$ и их производных конечного порядка. Локальность функционала $S(\Gamma)$ означает, что его вариационные производные

$$
\left(\delta_{A}\right)^{n} S(\Gamma)=\frac{\delta}{\delta \Gamma^{A_{1}}\left(x_{1}\right)} \cdots \frac{\delta}{\delta \Gamma^{A_{k}}\left(x_{k}\right)} S(\Gamma)
$$

являются квазилокальными обобшенными функциями. Символически это можно записать как

$$
\left(\delta_{A}\right)^{n} S(\Gamma) \sim \delta\left(x_{1}-x_{2}\right) \ldots \delta\left(x_{n-1}-x_{n}\right) .
$$

Функционалы $\Phi$ общего вида - это гладкие функции локальных функционалов

$$
\Phi(\Gamma)=f\left(S_{1}, S_{2}, \ldots\right)=f(S)
$$

обычно с определенной грассмановой четностью $\varepsilon(f)$. Будем называть такие функционалы мультилокальными функционалами. Производные по $S_{i}$ левые,

$$
\frac{\partial}{\partial S_{i}}=\partial_{i}, \quad \varepsilon\left(\partial_{i}\right)=\varepsilon(i)
$$


Ниже будут использоваться следующие сокрашенные обозначения:

$$
\begin{gathered}
\left(S_{i}\right)^{n}=S_{i_{1}} \ldots S_{i_{n}}, \quad \varepsilon\left(\left(S_{i}\right)^{n}\right)=\sum_{k=1}^{n} \varepsilon\left(i_{k}\right) \\
\left(\delta_{A} S_{i}\right)^{n}=\delta_{A_{1}} S_{i_{1}} \ldots \delta_{A_{n}} S_{i_{n}}, \quad \varepsilon\left(\left(\delta_{A} S_{i}\right)^{n}\right)=\sum_{k=1}^{n} \varepsilon_{A_{k}}+\sum_{k=1}^{n} \varepsilon\left(i_{k}\right) \\
\left(\delta_{A} \circ S_{i}\right)^{n}=\left(\delta_{A} S_{i}\right)^{n}(-1)^{\sum_{j=1}^{n-1} \varepsilon\left(i_{j}\right) \sum_{k=j+1}^{n} \varepsilon_{A_{k}}} \\
f_{i_{1} \ldots i_{n}}=\partial_{i_{1}} \ldots \partial_{i_{n}} f \\
(i)_{n}=i_{1} \ldots i_{n}, \quad \overleftarrow{(i)_{n}}=i_{n} \ldots i_{1}
\end{gathered}
$$

так что, например,

$$
f,{ }_{(i)_{n}}=f, i_{1} \ldots i_{n}, \quad f, \overleftarrow{(i)_{n}}=f, i_{n} \ldots i_{1}
$$

2.3. Локальные дифференциальные операторы $\nabla$. Определим на рассматриваемом пространстве функционалов мультилокальные дифференциальные операторы. Мультилокальность в данном случае означает, что образ оператора лежит в том же пространстве мультилокальных функционалов.

Начнем с однородных локальных дифференциальных операторов $\nabla_{n}$ произвольного порядка $n$. Рассмотрим локальное дифференциальное выражение

$$
\nabla_{n}=\int d x_{1} \ldots d x_{n} E_{n}^{A_{1} \ldots A_{n}}\left(x_{1}, \ldots, x_{n} ; \Gamma\right) \frac{\delta}{\delta \Gamma^{A_{1}}\left(x_{1}\right)} \ldots \frac{\delta}{\delta \Gamma^{A_{n}}\left(x_{n}\right)}=E_{n}^{(A)_{n}}\left(\delta_{A}\right)^{n},
$$

обладаюшее определенной грассмановой четностью $\varepsilon\left(\nabla_{n}\right)$, с коэффициентными функциями

$$
\begin{aligned}
E_{n}^{A_{1} \ldots A_{n}}\left(x_{1}, \ldots, x_{n} ; \Gamma\right)= & \sum_{m_{1} \ldots m_{n}} \int d x E_{n \mid m_{1} \ldots m_{n}}^{A_{1} \ldots A_{n}}\left(x, \Gamma(x), \partial_{\mu} \Gamma(x), \ldots\right) \times \\
& \times \prod_{k=1}^{n}\left(\partial_{\mu}\right)^{m_{k}} \delta\left(x-x_{k}\right),
\end{aligned}
$$

где $E_{n \mid m_{1} \ldots m_{n}}^{A_{1} \ldots A_{n}}\left(x, \Gamma(x), \partial_{\mu} \Gamma(x), \ldots\right)$ - гладкие функции своих аргументов. Коэффициентные функции $E_{n}^{A_{1} \ldots A_{n}}\left(x_{1}, \ldots, x_{n} ; \Gamma\right)=E_{n}^{(A)_{n}}$ - обобшенные функции по пространственным аргументам $x_{1}, \ldots, x_{n}$ с носителем в совпадающих точках $x_{1}=x_{2}=\cdots=x_{n}$. Следуя установившейся в теории поля традишии [4], будем называть такие обобшенные функции квазилокальными. Функции $E_{n}^{(A)_{n}}$ локально зависят от поля $\Gamma$, так что вариационные производные $\delta_{A_{n+1}} \ldots \delta_{A_{n+m}} E_{n}^{(A)_{n}}$ являются квазилокальными обобщенными функциями по всем пространственным аргументам $x_{1}, \ldots, x_{n}, x_{n+1}, \ldots, x_{n+m}$. Функции $E_{n}^{(A)_{n}}$ симметричны относительно одновременных перестановок индексов и координат

$$
\begin{aligned}
& E_{n}^{A_{1} \ldots A_{k} A_{k+1} \ldots A_{n}}\left(x_{1}, \ldots, x_{k}, x_{k+1}, \ldots, x_{n} ; \Gamma\right)= \\
& =(-1)^{\varepsilon_{A_{k}} \varepsilon_{A_{k+1}}} E_{n}^{A_{1} \ldots A_{k+1} A_{k} \ldots A_{n}}\left(x_{1}, \ldots, x_{k+1}, x_{k}, \ldots, x_{n} ; \Gamma\right)
\end{aligned}
$$


и обладают грассмановой четностью

$$
\varepsilon\left(E_{n}^{(A)_{n}}\right)=\varepsilon\left(\nabla_{n}\right)+\sum_{1}^{n} \varepsilon_{A_{k}}
$$

Оператор $\nabla_{n}$ на функционалах $f(S)$ определяется следуюшим образом:

$$
\left(\nabla_{n} f\right)(S)=E_{n}^{(A)_{n}}\left(\delta_{A} \circ S_{i}\right)^{n} f_{, \overleftarrow{(i)_{n}}}(S) \equiv S_{\nabla_{n}(i)_{n}} f_{, \overleftarrow{(i)_{n}}}(S)
$$

где

$$
S_{\nabla_{n}(i)_{n}}=S_{\nabla_{n}(i)_{n}}[S]=\frac{1}{n !} \nabla_{n}\left(S_{i}\right)^{n}=E_{n}^{(A)_{n}}\left(\delta_{A} \circ S_{i}\right)^{n}
$$

- производный от $S_{i_{1}}, \ldots, S_{i_{n}}$ локальный функционал с единым (мульти)индексом " $\nabla_{n}(i)_{n} "$, a

$$
\varepsilon\left(S_{\nabla_{n}(i)_{n}}\right)=\varepsilon\left(\nabla_{n}\right)+\sum_{k=1}^{n} \varepsilon\left(i_{k}\right) .
$$

Очевидно, что $\left(\nabla_{n} f\right)(S)$ есть функционал рассматриваемого вида. Разумеется, определение (5) распространяется и на тот случай, когда функционалы $S_{i}$ не обязательно локальны.

Общий локальный дифференциальный оператор $\nabla$ является линейной комбинацией операторов $\nabla_{n}$,

$$
\nabla=\sum_{n=0} c_{n} \nabla_{n}
$$

где $c_{n}$ - константы (не зависят от $x$ и $\Gamma$ ). $\nabla_{0}=S_{0}$ - произвольньй локальный функционал: формула (5) позволяет считать оператор умножения на локальный функционал дифференциальным оператором нулевого порядка. Ниже мы распространяем это определение на бесконечные суммы и оперируем в таком случае с формальными рядами, откладывая вопрос о смысле сходимости этих рядов.

Общий вид операторов, которые "хорошо" определены на рассматриваемом пространстве мультилокальных функционалов, - это произвольные гладкие функции $\omega(\nabla)=$ $\omega\left(\nabla_{n_{1}}, \nabla_{n_{2}}, \ldots\right)$ локальных операторов $\nabla_{n}$. Мы будем называть их мультилокальными операторами. Будем считать, что функции $\omega$ заданы в виде формальных рядов, что также определяет правило действия мультилокальных операторов на мультилокальные функционалы. Очевидно, что образ мультилокального оператора принадлежит пространству мультилокальных функционалов.

Вернемся к определению дифференциального оператора $\nabla_{n}$. Данное определение этого оператора по дифференциальному выражению (3) отличается от стандартного определения, задающего оператор $\Delta_{n}$ (ниже мы будем назьвать такие операторы каноническими дифференциальными операторами), тем дополнительным требованием, что "запрешается" действовать двум и более производным $\delta / \delta \Gamma^{A}(x)$ в одной и той же точке $x$ на один и тот же локальный функционал. Это требование эквивалентно правилу

$$
\frac{\delta}{\delta \Gamma^{A_{1}}(x)} \frac{\delta}{\delta \Gamma^{A_{2}}(x)} S(\Gamma)=0,
$$


т.е предписанию $\delta(0)=0$, в частности,

$$
\nabla_{n}\left(S_{i}\right)^{m}=0, \quad n \geqslant m+1
$$

Соотношение между операторами $\nabla_{n}$ и $\Delta_{n}$ можно пояснить также с точки зрения квазиклассического разложения. Давно замечено, что в квантовой теории появление величины $\delta(0)$ сопровождается дополнительной малостью по степеням постоянной Планка $\hbar$, параметра квазиклассического разложения (разложения по петлям). Соответственно оператор $\nabla_{n}$, отвечающий дифференциальному выражению (3), можно рассматривать как главное квазиклассическое приближение к каноническому дифференциальному оператору $\Delta_{n}$. Точньй смысл этого утверждения состоит в следующем. Произведем подстановки $S_{i} \rightarrow S_{i} / \hbar, f(S) \rightarrow f(S / \hbar)$ в функционалах, $\delta / \delta \Gamma \rightarrow \hbar \delta / \delta \Gamma$ в производных по полям и, наконец, подстановку

$$
\nabla_{n} \rightarrow \nabla_{n}(\hbar)=\frac{1}{\hbar} E_{n}^{(A)_{n}}\left(\hbar \delta_{A}\right)^{n}
$$

в дифференциальном выражении (3).

Чтобы нижеследуюшая формула имела смысл, необходимо отказаться от локальности либо дифференциальных операторов, регуляризовав их коэффициентные функции $E_{n}^{(A)_{n}}\left(x_{1}, \ldots, x_{n}\right)$, либо функционалов, считая функционалы $S_{i}$ регуляризованными нелокальными функционалами (можно, естественно, принять и то, и другое). Тогда нетрудно видеть, что для действия канонического оператора $\Delta_{n}(\hbar)$ на функционал $f(S / \hbar)$ имеем

$$
\Delta_{n}(\hbar) f\left(\frac{S}{\hbar}\right)=\left(\frac{1}{\hbar} S_{\nabla_{n}(i)_{n}}\right) f, \overleftarrow{(i)_{n}}\left(\frac{S}{\hbar}\right)+\sum_{k=1}^{n} \hbar^{k}\left(\frac{1}{\hbar} S_{\nabla_{n}(i)_{n-k}}\right) f, \overleftarrow{(i)_{n-k}}\left(\frac{S}{\hbar}\right)
$$

где функционалы

$$
S_{\nabla_{n}(i)_{n-k}}=E_{n}^{(A)_{k}(B)_{n-k}}\left(\delta_{A}\right)^{k}\left(\delta_{B} \circ S_{i}\right)^{n-k},
$$

так что

$$
\Delta_{n}(\hbar) f\left(\frac{S}{\hbar}\right)=\nabla_{n}(\hbar) f\left(\frac{S}{\hbar}\right)+O\left(\hbar, \frac{S}{\hbar}\right)
$$

и $O(\hbar, S / \hbar)$ как функция $S / \hbar$ имеет ненулевой порядок малости по $\hbar$. При снятии регуляризации функционалы $S_{\nabla_{n}(i)_{n-k}}(k \geqslant 1)$, а с ними и $O(\hbar, S / \hbar)$ становятся сингулярностями, пропорциональными $\delta(0)$.

Удобно сопоставить оператору $\nabla_{n}$ его символ $\widetilde{\nabla}_{n}=\widetilde{\nabla}_{n}(\Gamma, p)$, являющийся функционалом полей $\Gamma=\left\{\Gamma^{A}(x)\right\}$ и сопряженных им переменных $p=\left\{p_{A}(x)\right\}, \quad \varepsilon\left(p_{A}\right)=$ $\varepsilon\left(\Gamma^{A}\right)=\varepsilon_{A}$. Этот символ получается из дифференциального выражения (3) формальной заменой символа вариационной производной $\delta / \delta \Gamma^{A}(x)$ на переменную $p_{A}(x)$, т.е.

$$
\widetilde{\nabla}_{n}=\int d x_{1} \ldots d x_{n} E_{n}^{A_{1} \ldots A_{n}}\left(x_{1}, \ldots, x_{n} ; \Gamma\right) p_{A_{1}}\left(x_{1}\right) \ldots p_{A_{n}}\left(x_{n}\right)=E_{n}^{(A)_{n}}\left(p_{A}\right)^{n}
$$


2.4. Алгебра операторов. Рассматриваемое пространство функционалов замкнуто относительно действия мультилокальных операторов $\omega(\nabla)$, которые поэтому образуют ассоциативную алгебру.

Алгебра локальных операторов как ассоциативная алгебра не замкнута в себе. Произведение двух операторов $\nabla_{n} \nabla_{m}$ не есть локальный дифференциальный оператор типа $\nabla$. Действительно, результат действия (билокального) оператора $\nabla_{n} \nabla_{m}$ на функционал $f(S)$ будет содержать вторые вариационные производные локальных функционалов $S_{i}$ в разных точках, что невозможно при действии локального оператора типа $\nabla$.

Однако как алгебра Ли алгебра операторов $\nabla$ оказывается замкнутой, т.е. если операторы $\nabla^{(1)}, \nabla^{(2)}$ локальны, то их коммутатор

$$
\left[\nabla^{(1)}, \nabla^{(2)}\right]=\nabla^{(1)} \nabla^{(2)}-(-1)^{\varepsilon\left(\nabla^{(1)}\right) \varepsilon\left(\nabla^{(2)}\right)} \nabla^{(2)} \nabla^{(1)}
$$

оказывается тоже локальным дифференциальным оператором. Другими словами, можно сказать, что пространство локальных операторов замкнуто относительно операции $\mathrm{ad}$,

$$
\operatorname{ad} \nabla^{(1)}\left(\nabla^{(2)}\right)=\left[\nabla^{(1)}, \nabla^{(2)}\right]=\nabla^{(3)} .
$$

Достаточно убедиться в этом для однородных операторов $\nabla_{n}$. Если операторы

$$
\nabla_{m}=E_{m}^{(A)_{m}}\left(\delta_{A}\right)^{m}, \quad \nabla_{n}=E_{n}^{(A)_{n}}\left(\delta_{A}\right)^{n}
$$

локальны, то коммутатор

$$
\begin{aligned}
{\left[\nabla_{m}, \nabla_{n}\right]=} & m E_{m}^{C(A)_{m-1}} \delta_{C} E_{n}^{(B)_{n}}(-1)^{\varepsilon\left(E_{n}^{(B)_{n}}\right) \sum_{k=1}^{m-1} \varepsilon_{A_{k}}\left(\delta_{A}\right)^{m-1}\left(\delta_{B}\right)^{n}-} \\
& -(-1)^{\varepsilon\left(\nabla_{m}\right) \varepsilon\left(\nabla_{n}\right)} n E_{n}^{C(A)_{n-1}} \delta_{C} E_{m}^{(B)_{m}} \times \\
& \times(-1)^{\varepsilon\left(E_{m}^{(B)}\right)^{m}} \sum_{k=1}^{n-1} \varepsilon_{A_{k}}\left(\delta_{A}\right)^{n-1}\left(\delta_{B}\right)^{m} \equiv \\
\equiv & E_{m+n-1}^{([m, n])(A)_{m+n-1}}\left(\delta_{A}\right)^{m+n-1}=\nabla_{m+n-1}^{([m, n])}
\end{aligned}
$$

также локален. Действительно, определяемые предпоследним равенством коэффициентные функции $E_{m+n-1}^{([m, n])(A)_{m+n-1}}$ суть квазилокальные обобщенные функции по всем координатам $x_{1}, \ldots, x_{n+m-1}$ (поскольку они фактически являются свертками квазилокальных обобщенных функций). В классе функций, симметричных относительно одновременных перестановок координат и индексов, они определяются однозначно. Этот факт простым образом формулируется на языке символов $\widetilde{\nabla}$. Рассмотрим алгебру Ли функционалов $F(\Gamma, p)$, порождаемую канонической скобкой Пуассона. Если $F=F(\Gamma, p), \quad G=G(\Gamma, p)$, то

$$
\{F, G\}=\frac{\delta_{r} F}{\delta p_{A}} \frac{\delta G}{\delta \Gamma^{A}}-(-1)^{\varepsilon(F) \varepsilon(G)} \frac{\delta_{r} G}{\delta p_{A}} \frac{\delta F}{\delta \Gamma^{A}} .
$$

Здесь и ниже индекс $r$ при символе производной означает правую производную. Переменные Г и $p$ сопряжены именно в смысле этой скобки, т.е.

$$
\left\{p_{A}\left(x_{1}\right), \Gamma^{B}\left(x_{2}\right)\right\}=\delta_{A}^{B} \delta\left(x_{1}-x_{2}\right) .
$$

Нетрудно убедиться, что если $\widetilde{\nabla}_{m}, \widetilde{\nabla}_{n}$ - символы операторов $\nabla_{m}$ и $\nabla_{n}$, соответственно, а $\widetilde{\nabla}_{m+n-1}^{([m, n])}-$ символ их коммутатора, то

$$
\widetilde{\nabla}_{m+n-1}^{([m, n])}=\left\{\widetilde{\nabla}_{m}, \widetilde{\nabla}_{n}\right\} .
$$

На любые операторы $\nabla$ этот результат переносится по линейности. 


\section{3. ОПЕРАТОРЫ $\exp \nabla$ И ФУНКЦИОНАЛЫ $\exp S$}

В дальнейшем нам понадобятся мультилокальные операторы вида $\exp \nabla$, понимаемые как формальные ряды

$$
e^{\nabla}=\sum_{n=0} \frac{1}{n !} \nabla^{n}
$$

Значение этих операторов определяется тем, что с их помощью записываются многие преобразования в теории поля, в частности замена переменных $\Gamma \rightarrow \Gamma^{\prime}$ в функционалах.

Остановимся на свойствах этих операторов, вытекающих из только что доказанного факта замкнутости алгебры Ли операторов $\nabla$ или, эквивалентно, замкнутости множества этих операторов относительно операции аd: если $\nabla^{(1)}, \nabla^{(2)}$ - локальные дифференциальные операторы, то аd $\nabla^{(1)}\left(\nabla^{(2)}\right)=\left[\nabla^{(1)}, \nabla^{(2)}\right]$ также локальный дифференциальньй оператор. По индукции можно показать, что то же самое верно для операторов

$$
\left(\operatorname{ad} \nabla^{(1)}\right)^{n}\left(\nabla^{(2)}\right)=\underbrace{\left[\nabla^{(1)},\left[\nabla^{(1)}, \ldots,\left[\nabla^{(1)}, \nabla^{(2)}\right] \ldots\right]\right]}_{n}, \quad n=0,1,2, \ldots,
$$

а следовательно, и для оператора

$$
\begin{aligned}
\operatorname{Ad}\left(e^{\nabla^{(1)}}\right)\left(\nabla^{(2)}\right) & \equiv e^{\nabla^{(1)}} \nabla^{(2)} e^{-\nabla^{(1)}}=e^{\operatorname{ad} \nabla^{(1)}}\left(\nabla^{(2)}\right)= \\
& =\sum_{n=0} \frac{1}{n !}\left(\operatorname{ad} \nabla^{(1)}\right)^{n}\left(\nabla^{(2)}\right)=\nabla^{(3)}
\end{aligned}
$$

т.е. $\nabla^{(3)}$ есть локальный оператор. Другими словами, пространство локальных дифференциальных операторов замкнуто и относительно операции Ad. Кроме того, выполняется равенство

$$
e^{\nabla^{(1)}} e^{\nabla^{(2)}}=e^{\nabla^{(3)}}
$$

где $\nabla^{(3)}$ - локальный дифференциальный оператор. Этот факт является прямым следствием формулы Бейкера-Хаусдорфа-Дынкина [5],

$$
\nabla^{(3)}=\nabla^{(1)}+\nabla^{(2)}+\frac{1}{2}\left[\nabla^{(1)}, \nabla^{(2)}\right]+\cdots,
$$

где многоточие символизирует ряд по повторным коммутаторам $\nabla^{(1)}$ и $\nabla^{(2)}$. Другими словами, множество операторов вида $\exp \nabla$ замкнуто относительно ассоциативного умножения (и естественным образом определяет соответствуюшую ассоциативную алгебру).

В квантовой теории особую роль играют функционалы $f(S)=\exp S$, где $S$ - локальный функционал вида (1). Ниже мы ограничимся случаем, когда $S$ - бозевские функционалы, $\varepsilon(S)=0$. Для таких функционалов действие локального оператора $\nabla=$ $\sum_{n=0} c_{n} \nabla_{n}$ сводится к умножению на локальный функционал

$$
\nabla e^{S}=S_{\nabla}[S] e^{S}, \quad S_{\nabla}[S]=\sum_{n=0} c_{n} \frac{1}{n !} \nabla_{n} S^{n}=\sum_{n=0} c_{n} E_{n}^{(A) n}\left(\delta_{A} S\right)^{n}
$$


Оказывается, что множество функционалов этого вида замкнуто относительно действия операторов $\exp \nabla$ :

$$
e^{\nabla} e^{S}=e^{S^{\prime}}
$$

где $S^{\prime}$ - локальный функционал. Для доказателства рассмотрим однопараметрическое семейство операторов $\exp (\alpha \nabla)$, где $0 \leqslant \alpha \leqslant 1$. Тогда

$$
e^{\alpha \nabla} e^{S}=e^{S^{\prime}(\alpha)}
$$

где функционал $S^{\prime}(\alpha)$ гладко зависит от $\alpha, S^{\prime}(0)=S, S^{\prime}(1)=S^{\prime}$,

$$
S^{\prime}(\alpha)=S+\alpha S^{(1)}+\cdots=\sum_{m=0} \alpha^{m} S^{(m)}, \quad S^{(0)}=S .
$$

Для функционала (12) можно получить дифференциальное уравнение. Дифференцируя соотношение (11) по $\alpha$, имеем

$$
\nabla e^{S^{\prime}(\alpha)}=\frac{\partial S^{\prime}(\alpha)}{\partial \alpha} e^{S^{\prime}(\alpha)}
$$

Однако согласно формулам (9), справедливым и для не обязательно локальных функционалов, находим

$$
\begin{aligned}
\nabla e^{S^{\prime}(\alpha)} & =S_{\nabla}\left[S^{\prime}(\alpha)\right] e^{S^{\prime}(\alpha)} \\
S_{\nabla}\left[S^{\prime}(\alpha)\right] & =\sum_{n=0} c_{n} E_{n}^{(A)_{n}}\left(\delta_{A} S^{\prime}(\alpha)\right)^{n} .
\end{aligned}
$$

Окончательно получаем дифференциальное уравнение для $S^{\prime}(\alpha)$

$$
\frac{\partial S^{\prime}(\alpha)}{\partial \alpha}=S_{\nabla}\left[S^{\prime}(\alpha)\right]
$$

с начальным условием

$$
S^{\prime}(0)=S
$$

В классе гладких по $\alpha$ функционалов решение уравнения (13) при условии (14) единственно. Именно, подставляя в (13) разложение $(12)$, получаем для $S^{(m)}$ рекуррентные соотношения. В частности,

$$
S^{(1)}=S_{\nabla}[S]
$$

Интересно, что алгебра локальных операторов, в частности операция $\exp \nabla$, согласована с квазиклассическим разложением в следующем смысле. Сделаем подстановки

$$
\nabla \rightarrow \nabla(\hbar)=\sum_{n=0} c_{n} \nabla_{n}(\hbar)=\sum_{n=0} c_{n}\left(\frac{1}{\hbar} E_{n}^{(A)_{n}}\left(\hbar \delta_{A}\right)^{n}\right), \quad S \rightarrow \frac{1}{\hbar} S
$$

Рассмотрим тройку операторов $\nabla^{(i)}, i=1,2,3$. Тогда оказывается, что если операторы $\nabla^{(i)}$ связаны соотношением $(6)$, то то же верно и для операторов $\nabla^{(i)}(\hbar)$, т.е.

$$
\left[\nabla^{(1)}, \nabla^{(2)}\right]=\nabla^{(3)} \longrightarrow\left[\nabla^{(1)}(\hbar), \nabla^{(2)}(\hbar)\right]=\nabla^{(3)}(\hbar) .
$$


Достаточно в этом убедиться для однородных операторов $\nabla_{n}$. Как следствие, если $\nabla^{(i)}$ связаны соотношением (7) или (8), то то же верно и для $\nabla^{(i)}(\hbar)$ :

$$
\begin{aligned}
e^{\nabla^{(1)}} \nabla^{(2)} e^{-\nabla^{(1)}} & =\nabla^{(3)} \longrightarrow e^{\nabla^{(1)}(\hbar)} \nabla^{(2)}(\hbar) e^{-\nabla^{(1)}(\hbar)}=\nabla^{(3)}(\hbar), \\
e^{\nabla^{(1)}} e^{\nabla^{(2)}} & =e^{\nabla^{(3)}} \longrightarrow e^{\nabla^{(1)}(\hbar)} e^{\nabla^{(2)}(\hbar)}=e^{\nabla^{(3)}(\hbar)} .
\end{aligned}
$$

Легко также проверить, что если оператор $\nabla$ и функционалы $S$ и $S_{\nabla}[S]$ связаны соотношением (9), то аналогичная связь имеет место и для $\nabla(\hbar), S / \hbar, S \nabla / \hbar$ (с тем же самым $\left.S_{\nabla}\right)$, т.е.

$$
\nabla(\hbar) e^{\frac{1}{\hbar} S}=\frac{1}{\hbar} S_{\nabla}[S] e^{\frac{1}{\hbar} S} .
$$

Наконец, если оператор $\nabla$, функционалы $S$ и $S^{\prime}$ связаны соотношением (10), то справедливо соотношение

$$
e^{\nabla(\hbar)} e^{\frac{1}{\hbar} S}=e^{\frac{1}{\hbar} S^{\prime}},
$$

где функционал $S^{\prime}$ не зависит от $\hbar$. Доказательство повторяет доказательство формулы (10). При этом $\hbar$ сокращается в уравнении для $S^{\prime}(\alpha)$, которое тождественно совпадает с уравнением (13) при условии (14). Решение $S^{\prime}(\alpha)$ не зависит от $\hbar$.

\section{4. ЗАМЕНА ПЕРЕМЕННЫХ}

Мультилокальные функционалы $\Phi(\Gamma)$ и мультилокальные дифференциальные операторы $\omega(\nabla)$ допускают локальные замены переменных

$$
\Gamma^{A}=\Gamma^{A}(x) \longrightarrow \Gamma^{\prime A}=\Gamma^{A}\left(x, \Gamma(x), \partial_{\mu} \Gamma(x), \ldots\right),
$$

где $\Gamma^{\prime} A\left(x, \Gamma(x), \partial_{\mu} \Gamma(x), \ldots\right)$ - гладкие локальные функции полей $\Gamma(x)$ и их производных (до конечного порядка). Это означает, что $\delta \Gamma^{\prime A}(x) / \delta \Gamma^{B}(y)$ - квазилокальные обобшенные функции:

$$
\frac{\delta \Gamma^{\prime A}(x)}{\delta \Gamma^{B}(y)}=P_{B}^{A}\left(x, \Gamma(x), \partial_{\mu} \Gamma(x), \ldots, \partial_{\mu}\right) \delta(x-y),
$$

где $P_{B}^{A}\left(x, \Gamma(x), \partial_{\mu} \Gamma(x), \ldots, \partial_{\mu}\right)$ - полином по производным $\partial_{\mu}$ с коэффиициентами, являющимися локальными функциями полей $\Gamma(x)$ и их производных. Предполагается, что замена переменных сохраняет грассманову четность, $\varepsilon\left(\Gamma^{\prime A}\right)=\varepsilon\left(\Gamma^{A}\right)$. Примем также, что замена является обратимой (по крайней мере, пертурбативно по степеням производных полей Г), причем обратная замена

$$
\Gamma^{\prime A} \longrightarrow \Gamma^{A}=\Gamma^{A}\left(x, \Gamma^{\prime}(x), \partial_{\mu} \Gamma^{\prime}(x), \ldots\right)
$$

также является локальной,

$$
\frac{\delta \Gamma^{A}(x)}{\delta \Gamma^{\prime B}(y)}=Q_{B}^{A}\left(x, \Gamma^{\prime}(x), \partial_{\mu} \Gamma^{\prime}(x), \ldots, \partial_{\mu}\right) \delta(x-y) .
$$

Таким образом, локальные замены переменных образуют группу диффеоморфизмов, по крайней мере, в окрестности точки $\Gamma^{A}=0$. 
Локальные замены переменных естественным образом определяют локальные преобразования функционалов.

Примем, что функционалы $\Phi(\Gamma)$ преобразуются по скалярному (анти)представлению группы диффеоморфизмов, $\Phi^{\prime}(\Gamma)=\Phi\left(\Gamma^{\prime}\right)$. Очевидно, что если $S(\Gamma)$ представляет собой локальный функционал вида (1), то $S^{\prime}(\Gamma)$ также локален. Для мультилокальных функционалов общего вида (2) имеем

$$
\left(f^{\prime}(S)\right)(\Gamma)=\left(f\left(S^{\prime}\right)\right)(\Gamma)=(f(S))\left(\Gamma^{\prime}\right) .
$$

Таким образом, локальные преобразования переводят рассматриваемый класс функционалов в себя.

Соответственно локальные замены переменных индуцируют преобразования локальных дифференциальных операторов $\nabla$ :

$$
\nabla_{(\Gamma)} \longrightarrow \nabla_{(\Gamma)}^{\prime}=\nabla_{\left(\Gamma^{\prime}\right)},
$$

определяемые как

$$
(\nabla f(S))(\Gamma)=\nabla_{(\Gamma)} f(S(\Gamma)) \longrightarrow\left(\nabla^{\prime} f^{\prime}(S)\right)(\Gamma)=(\nabla f(S))\left(\Gamma^{\prime}\right)=\nabla_{\left(\Gamma^{\prime}\right)} f\left(S\left(\Gamma^{\prime}\right)\right) .
$$

Для дифференциального оператора $\nabla_{n}(3),(4)$ этот закон преобразования имеет вид

$$
\nabla_{n}^{\prime}=E_{n}^{\prime(A)_{n}}\left(\delta_{A}\right)^{n},
$$

где

$$
\begin{aligned}
E_{n}^{\prime A_{1} \ldots A_{n}}\left(x_{1}, \ldots, x_{n} ; \Gamma\right)= & \int d y_{1} \ldots d y_{n}(-1)^{\sum_{k=1}^{n-1} \varepsilon_{A_{k}} \sum_{i=k+1}^{n}\left(\varepsilon_{A_{i}}+\varepsilon_{B_{i}}\right)} \times \\
& \times E_{n}^{B_{1} \ldots B_{n}}\left(y_{1}, \ldots, y_{n} ; \Gamma^{\prime}\right) \frac{\delta \Gamma^{A_{1}}\left(x_{1}\right)}{\delta \Gamma^{B_{1}}\left(y_{1}\right)} \ldots \frac{\delta \Gamma^{A_{n}}\left(x_{n}\right)}{\delta \Gamma^{\prime B_{n}}\left(y_{n}\right)} .
\end{aligned}
$$

Коэффициентные функции $E_{n}^{A_{1} \ldots A_{n}}\left(x_{1}, \ldots, x_{n} ; \Gamma\right)$ преобразуются по тензорному (анти)представлению. Очевидно, что если $E_{n}^{A_{1} \ldots A_{n}}\left(x_{1}, \ldots, x_{n} ; \Gamma\right)$ - квазилокальная обобщенная функция, то $E_{n}^{\prime A_{1} \ldots A_{n}}\left(x_{1}, \ldots, x_{n} ; \Gamma\right)$ - также квазилокальная обобщенная функция (как свертка квазилокальных функций). Следовательно, оператор $\nabla_{n}^{\prime}$ является локальным. На произвольные операторы $\nabla=\sum c_{n} \nabla_{n}$ правило замены переменных переносится по линейности. Наконец, для мультилокальных операторов $\omega(\nabla)$ правило замены переменных имеет вид

$$
\omega(\nabla) \longrightarrow \omega\left(\nabla^{\prime}\right)
$$

Очевидно, что аналогичные свойства сохраняются, если определить преобразования функционалов и дифференциальных операторов при замене переменных по скалярному представлению

$$
\Phi^{\prime}\left(\Gamma^{\prime}\right)=\Phi(\Gamma), \quad \nabla_{\left(\Gamma^{\prime}\right)}^{\prime}=\nabla_{(\Gamma)} .
$$

Локальные замены переменных реализуются операторами $\exp \nabla_{1}$. Именно, имеют место формулы

$$
\Gamma^{\prime A}(x)=e^{\nabla_{1}} \Gamma^{A}(x)
$$


О ЛОКАЛЬНЫХ ОПЕРАТОРАХ В ВАРИАЦИОННЫХ ПРОИЗВОДНЫХ В ТЕОРИИ ПОЛЯ 267

с некоторым локальным дифференциальным оператором $\nabla_{1}$,

$$
S^{\prime}(\Gamma)=S\left(\Gamma^{\prime}\right)=e^{\nabla_{1}} S(\Gamma), \quad e^{\nabla_{1}} f(S)=f\left(S^{\prime}\right)
$$

Если $\Phi(\Gamma)$ рассматривается как оператор умножения в пространстве функционалов, то

$$
e^{\nabla_{1}} \Phi(\Gamma) e^{-\nabla_{1}}=\Phi^{\prime}(\Gamma)=\Phi\left(\Gamma^{\prime}\right)
$$

тогда как

$$
e^{\nabla_{1}} \frac{\delta}{\delta \Gamma^{A}(x)} e^{-\nabla_{1}}=\frac{\delta}{\delta \Gamma^{\prime A}(x)}=\int d y \frac{\delta \Gamma^{B}(y)}{\delta \Gamma^{A}(x)} \frac{\delta}{\delta \Gamma^{B}(y)}
$$

и соответственно

$$
e^{\nabla_{1}} \nabla e^{-\nabla_{1}}=\nabla^{\prime}
$$

\section{5. АНТИСКОБКА, МАСТЕР-УРАВНЕНИЕ, КАЛИБРОВОЧНЫЕ ПРЕОБРАЗОВАНИЯ}

5.1. Антискобка, канонические преобразования. Пусть $\nabla_{2}-$ локальный диффференциальный оператор второго порядка:

$$
\nabla_{2}=\frac{1}{2} \int d x_{1} d x_{2} E_{2}^{A_{1} A_{2}}\left(x_{1}, x_{2} ; \Gamma\right) \frac{\delta}{\delta \Gamma^{A_{1}}\left(x_{1}\right)} \frac{\delta}{\delta \Gamma^{A_{2}\left(x_{2}\right)}},
$$

грассмановой четности $1, \varepsilon\left(\nabla_{2}\right)=1$, нильпотентный, т.е.

$$
\nabla_{2}^{2}=\frac{1}{2}\left[\nabla_{2}, \nabla_{2}\right]=0
$$

Такой оператор определяет билинейную операцию на локальных функционалах, называемую антискобкой. Именно, если $S_{1}, S_{2}$ - любые локальные функционалы, то локальньй функционал - антискобка $\left(S_{1}, S_{2}\right)$ определяется как

$$
\begin{aligned}
&\left(S_{1}, S_{2}\right)=(-1)^{\varepsilon\left(S_{1}\right)} \nabla_{2}\left(S_{1} S_{2}\right)=2(-1)^{\varepsilon\left(S_{1}\right)} S_{\nabla_{2} 12}= \\
&=\int d x_{1} d x_{2} E_{2}^{A_{1} A_{2}}\left(x_{1}, x_{2} ; \Gamma\right)(-1)^{\left(\varepsilon_{A_{2}}+1\right) \varepsilon\left(S_{1}\right)} \frac{\delta S_{1}}{\delta \Gamma^{A_{1}}\left(x_{1}\right)} \frac{\delta S_{2}}{\delta \Gamma^{A_{2}\left(x_{2}\right)}} \\
& \varepsilon\left(\left(S_{1}, S_{2}\right)\right)=\varepsilon\left(S_{1}\right)+\varepsilon\left(S_{2}\right)+1 .
\end{aligned}
$$

Теперь можно написать (см. (5)), что

$$
\nabla_{2} f(S)=\frac{1}{2}(-1)^{\varepsilon\left(S_{i_{1}}\right)}\left(S_{i_{1}}, S_{i_{2}}\right) f_{, i_{2} i_{1}}(S) .
$$

Антискобку удобно представить в виде

$$
\left(S_{1}, S_{2}\right)=\int d x_{1} d x_{2} \frac{\delta_{r} S_{1}}{\delta \Gamma^{A_{1}}\left(x_{1}\right)} \widetilde{E}_{2}^{A_{1} A_{2}}\left(x_{1}, x_{2} ; \Gamma\right) \frac{\delta S_{2}}{\delta \Gamma^{A_{2}}\left(x_{2}\right)},
$$


где метрический тензор антискобки $\widetilde{E}_{2}^{A_{1} A_{2}}$ есть

$$
\widetilde{E}_{2}^{A_{1} A_{2}}=(-1)^{\varepsilon_{A_{1}}\left(\varepsilon_{A_{2}}+1\right)} E_{2}^{A_{1} A_{2}}=-(-1)^{\left(\varepsilon_{A_{1}}+1\right)\left(\varepsilon_{A_{2}}+1\right)} \widetilde{E}_{2}^{A_{2} A_{1}} .
$$

Очевидно, что антискобка обладает свойством антисимметрии

$$
\left(S_{1}, S_{2}\right)=-(-1)^{\left(\varepsilon\left(S_{1}\right)+1\right)\left(\varepsilon\left(S_{2}\right)+1\right)}\left(S_{2}, S_{1}\right) .
$$

Как следует из формул (19), (20), свойство нильпотентности (18) эквивалентно тождеству Якоби для антискобки, т.е.

$$
\nabla_{2}^{2}\left(S_{1} S_{2} S_{3}\right) \equiv 0 \longrightarrow(-1)^{\left(\varepsilon\left(S_{1}\right)+1\right)\left(\varepsilon\left(S_{3}\right)+1\right)}\left(S_{1},\left(S_{2}, S_{3}\right)\right)+\text { цикл }(1,2,3)=0 .
$$

В терминах коэффициентных функций $\widetilde{E}_{2}^{A_{1} A_{2}}$ это свойство означает (достаточно в (22) взять $\left.S_{1}=\Gamma^{A}(x), S_{2}=\Gamma^{B}(y), S_{3}=\Gamma^{C}(z)\right)$, что

$$
(-1)^{\left(\varepsilon_{A}+1\right)\left(\varepsilon_{C}+1\right)} \int d u \widetilde{E}_{2}^{A D}(x, u ; \Gamma) \frac{\delta}{\delta \Gamma^{D}(u)} \widetilde{E}_{2}^{B C}(y, z ; \Gamma)+\text { цикл }(A x, B y, C z)=0
$$

или в сокрашенных обозначениях

$$
(-1)^{\left(\varepsilon_{A}+1\right)\left(\varepsilon_{C}+1\right)} \widetilde{E}_{2}^{A D} \delta_{D} \widetilde{E}_{2}^{B C}+\text { цикл }(A, B, C)=0 .
$$

Конечно, справедливо и обратное. Если тензор $\widetilde{E}_{2}^{A_{1} A_{2}}$ удовлетворяет условию антисимметрии (21) и тождеству Якоби (23), то оператор $\nabla_{2}(17)$ с тензором $E_{2}^{A_{1} A_{2}}$, определяемым соотношением (21), является нильпотентным.

Стандартно полагается, что при замене переменных $\Gamma \rightarrow \Gamma^{\prime}$ антискобка преобразуется, $(.,.) \rightarrow(., .)^{\prime}$ (штрих при скобке есть символ новой антискобки), по следуюшему правилу:

$$
\left(S_{1}, S_{2}\right)(\Gamma) \longrightarrow\left(S_{1}, S_{2}\right)\left(\Gamma^{\prime}\right)=\left(S_{1}^{\prime}, S_{2}^{\prime}\right)^{\prime}(\Gamma)=\delta_{r A} S_{1}^{\prime} \widetilde{E}_{2}^{\prime A B} \delta_{B} S_{2}^{\prime}(\Gamma),
$$

откуда следует, что метрический тензор антискобки преобразуется по тензорному (анти)представлению

$$
\widetilde{E}_{2}^{\prime A_{1} A_{2}}\left(x_{1}, \ldots, x_{n} ; \Gamma\right)=\int d y_{1} d y_{2} \frac{\delta_{r} \Gamma^{A_{1}}\left(x_{1}\right)}{\delta \Gamma^{\prime B_{1}}\left(y_{1}\right)} \widetilde{E}_{2}^{B_{1} B_{2}}\left(y_{1}, y_{2} ; \Gamma^{\prime}\right) \frac{\delta \Gamma^{A_{2}}\left(x_{2}\right)}{\delta \Gamma^{\prime B_{2}}\left(y_{2}\right)} .
$$

Эти трансформационные свойства антискобки согласуются с правилами, получаемыми из определения (19) антискобки $\left(S_{1}, S_{2}\right)=(-1)^{\varepsilon\left(S_{1}\right)} \nabla_{2}\left(S_{1} S_{2}\right), \quad\left(S_{1}, S_{2}\right)^{\prime}=$ $(-1)^{\varepsilon\left(S_{1}\right)} \nabla_{2}^{\prime}\left(S_{1} S_{2}\right)$ и трансформационных свойств $(15)$ операторов $\nabla$.

В связи с антискобкой (а также в связи с мастер-уравнением, см. ниже) возникает вопрос о преобразованиях полей $\Gamma \rightarrow \Gamma^{\prime}=e^{\nabla_{1}} \Gamma$, которые ее сохраняют (локальных канонических заменах переменных):

$$
\left(S_{1}, S_{2}\right)^{\prime}(\Gamma)=\left(S_{1}, S_{2}\right)(\Gamma)
$$


На языке операторов это означает, что

$$
e^{\nabla_{1}} \nabla_{2} e^{-\nabla_{1}}=\nabla_{2}
$$

или

$$
\left[e^{\nabla_{1}}, \nabla_{2}\right]=0,
$$

т.е. преобразование сохраняет оператор $\nabla_{2}$. На языке коэффициентных функций это означает, что

$$
\widetilde{E}_{2}^{\prime A_{1} A_{2}}\left(x_{1}, x_{2} ; \Gamma\right)=\widetilde{E}_{2}^{A_{1} A_{2}}\left(x_{1}, x_{2} ; \Gamma\right) .
$$

Класс таких преобразований существенно зависит от свойств коэффициентных функции $\widetilde{E}_{2}^{A_{1} A_{2}}$ (например, в тривиальном случае $\widetilde{E}_{2}^{A_{1} A_{2}}=0$ он включает вообше все преобразования). В конечномерном случае, когда коэффициентные функции суть конечномерные матрицы $\widetilde{E}_{2}^{i j}$, этот класс преобразований определяется рангом матрицы $\widetilde{E}_{2}^{i j}$.

Легко видеть, что свойство (24) удовлетворяется, если

$$
\nabla_{1}=\left[\nabla_{2}, F\right]=\nabla_{2} F+F \nabla_{2}=-\int d x_{1} d x_{2} \frac{\delta_{r} F}{\delta \Gamma^{A_{1}}\left(x_{1}\right)} \widetilde{E}_{2}^{A_{1} A_{2}}\left(x_{1}, x_{2} ; \Gamma\right) \frac{\delta}{\delta \Gamma^{A_{2}}\left(x_{2}\right)},
$$

где $F$ - локальный фермионньй функционал, $\varepsilon(F)=1$. Это следует из легко проверяемого соотношения $\left[\nabla_{1}, \nabla_{2}\right]=0$. Очевидно, что $\nabla_{1} S=(S, F)$. Таким образом, преобразование

$$
\Gamma^{A} \rightarrow \Gamma^{\prime A}=e^{\left[\nabla_{2}, F\right]} \Gamma^{A}
$$

является каноническим.

В конечномерном случае можно показать, что если матрица $\widetilde{E}_{2}^{i j}$ невырожденна, то преобразования, порождаемые оператором $e^{\left[\nabla_{2}, F\right]}$, исчерпьвают все преобразования, сохраняюшие антискобку или, что то же самое, оператор $\nabla_{2}$.

5.2. Мастер-уравнение, калибровочные преобразования. Нильпотентный локальный оператор $\nabla_{2}, \varepsilon\left(\nabla_{2}\right)=1$, определяет на локальных функционалах $S(\Gamma)$ мастер-уравнение

$$
\nabla_{2} e^{\frac{i}{\hbar} S}=0
$$

или

$$
(S, S)=\nabla_{2} S^{2}=0 .
$$

Оно нетривиально только для бозевских функционалов.

Очевидно, что это уравнение инвариантно относительно канонических преобразований функционалов, индуцированных локальными каноническими преобразованиями полей $\Gamma$, сохраняюшими антискобку. В частности, если $S$ - решение мастер-уравнения, то

$$
S^{\prime}=e^{\left[\nabla_{2}, F\right]} S, \quad \varepsilon(F)=1,
$$

также решение. Преобразование (27) можно представить как

$$
e^{\frac{i}{\hbar} S} \longrightarrow e^{\frac{i}{\hbar} S^{\prime}}=e^{\left[\nabla_{2}, F\right]} e^{\frac{i}{\hbar} S} .
$$


Инвариантность мастер-уравнения, как и антискобки, относительно канонических преобразований (28) есть следствие того, что для нильпотентного оператора $\nabla_{2}$ оператор $\left[\nabla_{2}, F\right]$ коммутирует с $\nabla_{2}$. С другой стороны, любой оператор вида $\left[\nabla_{2}, \nabla\right]$ с произвольным $\nabla$ коммутирует с $\nabla_{2}$. Отсюда следует, что любое преобразование вида

$$
e^{\frac{i}{\hbar} S} \longrightarrow e^{\frac{i}{\hbar} S^{\prime}}=e^{\left[\nabla_{2}(\hbar), \nabla(\hbar)\right]} e^{\frac{i}{\hbar} S}
$$

с произвольным локальным оператором $\nabla, \varepsilon(\nabla)=1$, переводит локальное решение $S$ мастер-уравнения в локальное решение $S^{\prime}$ (не зависяшее от $\hbar$ ). Преобразования вида (29) будем называть калибровочными преобразованиями. Заметим, что представить калибровочное преобразование в виде оператора, действующего непосредственно на $S$, довольно сложно. Замечательно также, что операторы калибровочных преобразований $e^{\left[\nabla_{2}, \nabla\right]}$ образуют ассоциативную алгебру.

Оказьвается, однако, что на решениях мастер-уравнения любое калибровочное преобразование (29) сводится к каноническому преобразованию (28) с некоторым локальным фермиевским функционалом $F(\Gamma)$. Тот факт, что функционал $F$ существует, можно доказать, например, следующим образом. Вместо оператора $\nabla$ рассмотрим оператор $\alpha \nabla$. Тогда соотношение

$$
e^{\alpha\left[\nabla_{2}(\hbar), \nabla(\hbar)\right]} e^{\frac{i}{\hbar} S}=e^{\left[\nabla_{2}, F(\alpha)\right]} e^{\frac{i}{\hbar} S}
$$

будет выполняться, если, например, $F(\alpha)$ является решением уравнения

$$
\int_{0}^{1} d \beta e^{\beta\left[\nabla_{2}, F(\alpha)\right]} \frac{\partial F(\alpha)}{\partial \alpha}=S_{\nabla}(\alpha), \quad F(0)=0
$$

а локальный, не зависящий от $\hbar$, фермиевский функционал $S_{\nabla}(\alpha)$ определяется из соотношения

$$
\hbar \nabla(\hbar) e^{\alpha\left[\nabla_{2}(\hbar), \nabla(\hbar)\right]} e^{\frac{i}{\hbar} S}=S_{\nabla}(\alpha) e^{\alpha\left[\nabla_{2}(\hbar), \nabla(\hbar)\right]} e^{\frac{i}{\hbar} S} .
$$

Пертурбативно по $\alpha$ формальное локальное решение уравнения (30) для $F(\alpha)$ сушествует.

Вопрос о том, когда калибровочные преобразования действуют на решениях транзитивно, определяется свойствами оператора $\nabla_{2}$, т.е. его коэффициентных функций $E_{2}^{A_{1} A_{2}}$, и дополнительными условиями на $S$ (ясно, что $S=0$ всегда является решением).

В рамках стандартной БВ-формулировки калибровочных теорий поля метрика $\widetilde{E}_{2}^{A_{1} A_{2}}$ является неособенной, а поля Г представляют собой координаты Дарбу для метрики:

$$
\begin{gathered}
\Gamma^{A}(x)=\left\{\Phi^{a}(x), \Phi_{a}^{*}(x)\right\}, \quad \varepsilon\left(\Phi_{a}^{*}\right)=\varepsilon\left(\Phi^{a}\right)+1, \\
\nabla_{2}=\int d x(-1)^{\varepsilon\left(\Phi^{a}\right)} \frac{\delta}{\delta \Phi^{a}(x)} \frac{\delta}{\delta \Phi_{a}^{*}(x)} .
\end{gathered}
$$

Поля $\Phi^{a}$ подразделяются на две группы: $\Phi^{a}=\left\{\varphi^{i}, C^{\alpha}\right\}$, где $\varphi^{i}-$ поля исходной классической теории, $C^{\alpha}$ - гостовские поля. Аналогичным образом подразделяются и поля $\Phi_{a}^{*}$ (называемые антиполями). Всем переменным приписывается гостовское число gh: 
$\operatorname{gh}\left(\varphi^{i}\right)=0, \operatorname{gh}\left(C^{\alpha}\right)=1, \operatorname{gh}\left(\Phi_{a}^{*}\right)=-\operatorname{gh}\left(\Phi^{a}\right)-1$. Решение $S$ мастер-уравнения (26) ишется в виде ряда по $C$ :

$$
S=\mathcal{S}+\sum_{n=1} S^{(n)}, \quad S^{(n)}=O\left(C^{n}\right), \quad \varepsilon(S)=\operatorname{gh}(S)=0,
$$

где $\mathcal{S}$ - локальное действие классической теории. Предполагается, что действие $\mathcal{S}$ обладает калибровочной симметрией, т.е. удовлетворяет калибровочным тождествам

$$
R_{\alpha}^{i}\left(x, \varphi(x), \partial_{\mu} \varphi(x), \ldots, \partial_{\mu}\right) \frac{\delta \mathcal{S}}{\delta \varphi^{i}(x)}=0
$$

где $R_{\alpha}^{i}\left(x, \varphi(x), \partial_{\mu} \varphi(x), \ldots, \partial_{\mu}\right)$ - полином по $\partial_{\mu}$ с локальными по координатам $x$ коэффициентами. Кроме того, действие $\mathcal{S}$ удовлетворяет некоторым условиям, так называемым условиям регулярности (см. [6] и цитированную там литературу), смысл которых состоит в том, что среди произвольного набора из экстремалей $\delta \mathcal{S} / \delta \varphi^{i}(x)$ и их производных по координатам можно выделить линейно (с локальными коэффициентами) независимые и линейно зависимые элементы, а единственными связями между ними являются линейные комбинации (с локальными коэффициентами) калибровочных тождеств и их производных по координатам. При выполнении условий регулярности доказано [6], что мастер-уравнение имеет локальные решения вида (32), а произвол в решении описывается каноническими преобразованиями (27), т.е. канонические преобразования действуют на пространстве решений типа (32) транзитивно.

Сравним вьшеприведенные выражения с аналогичными формальными выражениями канонического БВ-формализма [1]. Аналогом нильпотентного оператора $\nabla_{2}$ является канонический дифференшиальный оператор второго порядка $\Delta_{2}$ (мы ограничимся случаем, когда переменные $\Gamma^{A}(x)$ - координаты Дарбу для метрики $E_{2}^{A_{1} A_{2}}$, т.е. дифференциальные выражения для $\nabla_{2}$ и $\Delta_{2}$ имеют вид (31)). Формально действие в квантовой теории поля должно удовлетворять квантовому мастер-уравнению

$$
\Delta_{2} e^{\frac{i}{\hbar} S}=0
$$

или

$$
\frac{1}{2}(S, S)=i \hbar \Delta_{2} S
$$

Уравнение (26) и формальное уравнение (33) отличаются слагаемыми, пропорциональными $\hbar \delta(0)$, т.е. мастер-уравнение (26) является квазиклассическим приближением квантового мастер-уравнения (33), а решения мастер-уравнения (26) являются нулевыми по $\hbar$ (квазиклассическими) приближениями решений квантового мастер-уравнения (33).

Формальный оператор калибровочных преобразований есть

$$
e^{\left[\Delta_{2}, F\right]}=D e^{\left[\nabla_{2}, F\right]}=e^{\frac{i}{\hbar}(-i \hbar \ln D)} e^{\left[\nabla_{2}, F\right]},
$$

где

$$
D=\frac{D\left(\Phi^{\prime}\right)}{D(\Phi)}=\left(\frac{D\left(\Gamma^{\prime}\right)}{D(\Gamma)}\right)^{1 / 2}, \quad \Gamma^{\prime A}=e^{\left[\nabla_{2}, F\right]} \Gamma^{A}
$$


При этом функционал $S^{\prime}$, связанный с функционалом $S$ соотношением

$$
e^{\frac{i}{\hbar} S^{\prime}}=e^{\left[\Delta_{2}, F\right]} e^{\frac{i}{\hbar} S}
$$

есть решение квантового мастер-уравнения, если таковым является $S$.

Таким образом, оператор $\exp \left(\left[\nabla_{2}, F\right]\right)$ - квазиклассическое приближение формального оператора (34).

Отметим также следуюший факт. Обычно на решение квантового мастер-уравнения (33) накладываются граничные условия

$$
\left.S\right|_{\hbar=0, C=0}=\mathcal{S}
$$

В этом случае операторы вида (34) хотя и переводят решение квантового мастер-уравнения в решение, однако не действуют на решениях транзитивно [7].

\section{6. $S p(2)$-МАСТЕР-УРАВНЕНИЕ}

В этом разделе мы рассмотрим $S p(2)$-мастер-уравнение [8], описываюшее $S p(2)$-симметричное обобшение БВ-формализма. Полный набор переменных теории $\Gamma^{\Sigma}$ (в этом разделе мы слегка изменяем обозначения индексов у переменных Г) разбивается на группы $\Phi^{A}, \Phi_{A a}^{*}, \bar{\Phi}_{A}, a, b, c=1,2$, каждая из переменных является функцией координат $x^{\mu}$. Переменные $\Phi^{A}$, в свою очередь, разбиты на группы $\Phi^{A}=\left(\varphi^{i}, C^{\alpha b}, B^{\alpha}\right)$, где $\varphi^{i}$ - переменные классической теории, $C^{\alpha b}$ - поля гостов и антигостов, $B^{\alpha}-$ поля, вводяшие калибровку. Всем полям приписывается грассманова четность $\varepsilon\left(\Phi^{A}\right)=\varepsilon\left(\bar{\Phi}_{A}\right)=$ $\varepsilon_{A}, \quad \varepsilon\left(\Phi_{A a}^{*}\right)=\varepsilon_{A}+1, \quad \varepsilon\left(B^{\alpha}\right)=\varepsilon_{\alpha}, \quad \varepsilon\left(C^{\alpha b}\right)=\varepsilon_{\alpha}+1$ и новое гостовское число ngh: $\operatorname{ngh}(\varphi)=0, \operatorname{ngh}\left(C^{b}\right)=1, \operatorname{ngh}(B)=2, \operatorname{ngh}(\bar{\Phi})=-2-\operatorname{ngh}(\Phi), \operatorname{ngh}\left(\Phi_{a}^{*}\right)=-1-\operatorname{ngh}(\Phi)$, $\operatorname{ngh}(F M)=\operatorname{ngh}(F)+\operatorname{ngh}(M)$.

В $S p(2)$-формализме эффективное действие $S\left(\Phi, \Phi_{a}^{*}, \bar{\Phi}\right), \operatorname{ngh}(S)=\varepsilon(S)=0$, удовлетворяет $S p(2)$-мастер-уравнению

$$
\begin{gathered}
\frac{1}{2}(S, S)^{a}+\int d x \varepsilon^{a b} \Phi_{A b}^{*}(x) \frac{\delta}{\delta \bar{\Phi}(x)_{A}} S=0, \\
\varepsilon^{a b}=-\varepsilon^{b a}, \quad \varepsilon_{a b} \varepsilon^{b c}=\delta_{a}^{c}, \quad \varepsilon_{12}=\varepsilon^{21}=1,
\end{gathered}
$$

и начальному условию

$$
\left.S\right|_{\Phi_{a}^{*}=\bar{\Phi}=0}=\mathcal{S}(\varphi),
$$

где $\mathcal{S}(\varphi)$ - исходное классическое действие, обладаюшее калибровочной симметрией $R_{\alpha}^{i} \delta \mathcal{S} / \delta \varphi^{i}=0$. В формуле (35) $(.,)^{a}$ означает дублет антискобок:

$$
\left(S_{1}, S_{2}\right)^{a} \equiv \int d x\left(\frac{\delta_{r} S_{1}}{\delta \Phi^{A}(x)} \frac{\delta S_{2}}{\delta \Phi_{A a}^{*}(x)}-\frac{\delta_{r} S_{1}}{\delta \Phi_{A a}^{*}(x)} \frac{\delta S_{2}}{\delta \Phi^{A}(x)}\right) .
$$

Введем дублет операторов

$$
\begin{array}{rlrl}
\bar{\nabla}^{a} & =\nabla_{2}^{a}+\nabla_{1}^{a}, & & \varepsilon\left(\bar{\nabla}^{a}\right)=1, \\
\nabla_{2}^{a}=\int d x(-1)^{\varepsilon} A \frac{\delta}{\delta \Phi^{A}(x)} \frac{\delta}{\delta \Phi_{A a}^{*}(x)}, & & \varepsilon\left(\nabla_{2}^{a}\right)=1, \\
\nabla_{1}^{a}=i \int d x \varepsilon^{a b} \Phi_{A b}^{*}(x) \frac{\delta}{\delta \bar{\Phi}_{A}(x)}, & \varepsilon\left(\nabla_{1}^{a}\right)=1 .
\end{array}
$$


Эти операторы нильпотентны:

$$
\begin{gathered}
\nabla_{2}^{a} \nabla_{2}^{b}+\nabla_{2}^{b} \nabla_{2}^{a}=0, \quad \nabla_{1}^{a} \nabla_{1}^{b}+\nabla_{1}^{b} \nabla_{1}^{a}=0, \quad \nabla_{2}^{a} \nabla_{1}^{b}+\nabla_{2}^{b} \nabla_{1}^{a}+\nabla_{1}^{a} \nabla_{2}^{b}+\nabla_{1}^{b} \nabla_{2}^{a}=0 \\
\bar{\nabla}^{a} \bar{\nabla}^{b}+\bar{\nabla}^{b} \bar{\nabla}^{a}=0 .
\end{gathered}
$$

Введенный выше дублет антискобок связан с дублетом операторов $\nabla_{2}^{a}$ соотношением

$$
\left(S_{1}, S_{2}\right)^{a}=(-1)^{\varepsilon\left(S_{1}\right)} \nabla_{2}^{a}\left(S_{1} S_{2}\right) .
$$

С использованием операторов $\bar{\nabla}^{a} S p(2)$-мастер-уравнение записывается в следующей эквивалентной форме:

$$
\bar{\nabla}^{a}(\hbar) e^{\frac{i}{\hbar} S}=0 .
$$

При тех же предположениях, что и в п. 5.2, в статье [9] было доказано, что $S p(2)$-мастер-уравнение имеет локальные решения и найден произвол обшего решения. Мы покажем, что рассматриваемый здесь формализм является естественным аппаратом для этой цели.

Введем класс операторов, которые мы будем называть операторами калибровочных преобразований:

$$
K=e^{U}, \quad U=\frac{1}{2} \varepsilon_{a b}\left[\bar{\nabla}^{b},\left[\bar{\nabla}^{a}, \nabla\right]\right], \quad \varepsilon(U)=0,
$$

где $\nabla$ - произвольный локальный оператор, $\varepsilon(\nabla)=0$. Очевидно, что оператор $U$ обладает свойством

$$
\left[\bar{\nabla}^{a}, U\right]=0,
$$

и, как следствие, оператор калибровочного преобразования коммутирует с операторами $\bar{\nabla}^{a}$, т.е.

$$
\left[\bar{\nabla}^{a}, K\right]=\left[\bar{\nabla}^{a}, e^{U}\right]=0 .
$$

Из формулы (38) следует, что операторы вида $U$ образуют алгебру Ли:

$$
\left[U^{(1)}, U^{(2)}\right]=\frac{1}{2} \varepsilon_{a b}\left[\bar{\nabla}^{b},\left[\bar{\nabla}^{a}, \nabla_{3}\right]\right]=U^{(3)}, \quad \nabla_{3}=\left[U^{(1)}, \nabla^{(2)}\right] .
$$

По формуле Бейкера-Хаусдорфа-Дынкина получаем, что

$$
e^{U^{(1)}} e^{U^{(2)}}=e^{U^{(3)}} .
$$

Следовательно, операторы калибровочных преобразований образуют ассоциативную алгебру.

Из результатов раздела 3 следует, что если $S$ - локальный функционал, не зависяший от $\hbar$, то и функционал $S^{\prime}$, определенный равенством

$$
e^{\frac{i}{\hbar} S^{\prime}}=K(\hbar) e^{\frac{i}{\hbar} S}
$$

4 Теоретическая и математическая физика, т. 120, № 2, 1999 г. 
где

$$
K(\hbar)=e^{U(\hbar)}, \quad U(\hbar)=\frac{1}{2} \varepsilon_{a b}\left[\bar{\nabla}^{b}(\hbar),\left[\bar{\nabla}^{a}(\hbar), \nabla(\hbar)\right]\right]
$$

является локальным функционалом и не зависит от $\hbar$. Кроме того, если функционал $S$ удовлетворяет $S p(2)$-мастер-уравнению (35), то в силу свойства (39) функционал $S^{\prime}$ также удовлетворяет $S p(2)$-мастер-уравнению:

$$
\bar{\nabla}^{a}(\hbar) e^{\frac{i}{\hbar} S^{\prime}}=0
$$

Для функционалов $S$, удовлетворяющих $S p(2)$-мастер-уравнению, справедливо соотношение

$$
e^{\frac{1}{2} \varepsilon_{a b}\left[\bar{\nabla}^{b}(\hbar),\left[\bar{\nabla}^{a}(\hbar), \nabla(\hbar)\right]\right]} e^{\frac{i}{\hbar} S}=e^{\frac{1}{2} \varepsilon_{a b}\left[\bar{\nabla}^{b}(\hbar),\left[\bar{\nabla}^{a}(\hbar), \nabla_{0}(\hbar)\right]\right]} e^{\frac{i}{\hbar} S}
$$

где

$$
\nabla_{0}(\hbar)=\frac{1}{\hbar} Y
$$

$Y$ - некоторый локальный функционал, не зависящий от $\hbar, \varepsilon(Y)=0$. Действительно, если вместо оператора $\nabla$ рассмотреть оператор $\alpha \nabla$, то можно показать, что соотношение

$$
e^{\frac{i}{\hbar} S^{\prime}(\alpha)}=e^{\frac{\alpha}{2} \varepsilon_{a b}\left[\bar{\nabla}^{b}(\hbar),\left[\bar{\nabla}^{a}(\hbar), \nabla(\hbar)\right]\right]} e^{\frac{i}{\hbar} S}=e^{\frac{1}{2} \varepsilon_{a b}\left[\bar{\nabla}^{b}(\hbar),\left[\bar{\nabla}^{a}(\hbar), \frac{1}{\hbar} Y(\alpha)\right]\right]} e^{\frac{i}{\hbar} S}
$$

будет выполнено, например, для функционала $Y(\alpha)$, удовлетворяюшего уравнению

$$
\begin{gathered}
\int_{0}^{1} d \beta e^{\beta \frac{1}{2} \varepsilon_{a b}\left[\bar{\nabla}^{b}(\hbar),\left[\bar{\nabla}^{a}(\hbar), \frac{1}{\hbar} Y(\alpha)\right]\right]} \frac{\partial Y(\alpha)}{\partial \alpha} e^{-\beta \frac{1}{2} \varepsilon_{a b}\left[\bar{\nabla}^{b}(\hbar),\left[\bar{\nabla}^{a}(\hbar), \frac{1}{\hbar} Y(\alpha)\right]\right]} e^{\frac{i}{\hbar} S^{\prime}(\alpha)}= \\
=\nabla(\hbar) e^{\frac{i}{\hbar} S^{\prime}(\alpha)}=\frac{i}{\hbar} S_{\nabla}(\alpha) e^{\frac{i}{\hbar} S^{\prime}(\alpha)}, \quad Y(0)=0
\end{gathered}
$$

пертурбативное по $\alpha$ решение которого сушествует. Таким образом, если рассматривается действие операторов калибровочных преобразований (37) на функционалы $\exp \left(\frac{i}{\hbar} S\right)$, где $S$ удовлетворяет $S p(2)$-мастер-уравнению, то можно ограничиться операторами $K$ с $\nabla=\nabla_{0}$.

Заметим, что любая замена полей

$$
\Phi^{A} \rightarrow \Phi^{\prime A}=\Phi^{\prime A}(\Phi)=e^{E_{1}^{B}(\Phi) \delta_{B}} \Phi^{A}
$$

может быть расширена до калибровочного преобразования в следуюшем смысле:

$$
\begin{gathered}
\left.e^{\frac{i}{2} \varepsilon_{a b}\left[\bar{\nabla}_{b},\left[\bar{\nabla}^{a}, \bar{\Phi}_{A} T^{A}\right]\right]} G(\Gamma)\right|_{\Phi_{a}^{*}=\bar{\Phi}=0}=e^{T^{A} \delta_{A}} g(\Phi)=g\left(\Phi^{\prime}\right), \\
g(\Phi)=\left.G(\Gamma)\right|_{\Phi^{*}=\bar{\Phi}=0} .
\end{gathered}
$$

Перейдем к описанию произвола в решении $S p(2)$-мастер-уравнения. Пусть

$$
S=\mathcal{S}+\sum_{n=1} S_{(n)}, \quad S_{(n)} \sim C^{k} B^{n-k}
$$


- решение $S p(2)$-мастер-уравнения с

$$
S_{(1)}=\int d x\left(C^{\alpha a} R_{\alpha}^{i} \varphi_{i a}^{*}+\varepsilon^{a b} C_{\alpha b \mid a}^{*} B^{\alpha}+B^{\alpha} R_{\alpha}^{i} \bar{\varphi}_{i}(-1)^{\varepsilon_{i}+\varepsilon_{\alpha}}\right) .
$$

В статье [9] было показано, что если два решения $S$ и $S_{1}$ совпадают с точностью до $n$-го порядка разложения в ряд по переменным $C^{\alpha b}, B^{\alpha}$, то их разность $\delta S_{(n+1)}$ в $(n+$ 1)-приближении

$$
S_{1}-S=\delta S_{(n+1)}+O\left(C^{k} B^{n+2-k}\right), \quad \delta S_{(n+1)} \sim C^{k} B^{n+1-k},
$$

может быть представлена в виде

$$
\begin{gathered}
\delta S_{(n+1)}=\frac{1}{2} \varepsilon_{a b} \omega^{b} \omega^{a} X_{(n+1)}, \quad X_{(n+1)} \sim C^{k} B^{n+1-k}, \\
\omega^{a}=\int d x\left((-1)^{\varepsilon_{i}} L_{i} \frac{\delta}{\delta \varphi_{i a}^{*}}-(-1)^{\varepsilon_{\alpha}} R_{\alpha}^{i} \varphi_{i b}^{*} \frac{\delta}{\delta C_{\alpha b \mid a}^{*}}+\right. \\
\left.+\left((-1)^{\varepsilon_{i}} R_{\alpha}^{i} \bar{\varphi}_{i}+\varepsilon^{c b} C_{\alpha b \mid c}^{*}\right) \frac{\delta}{\delta B_{\alpha a}^{*}}-(-1)^{\varepsilon_{\alpha}} \varepsilon^{a b} B^{\alpha} \frac{\delta}{\delta C^{\alpha b}}+\varepsilon^{a b} \Phi_{A b}^{*} \frac{\delta}{\delta \bar{\Phi}_{A}}\right), \\
L_{i}(x) \equiv \frac{\delta \mathcal{S}}{\delta \varphi^{i}(x)},
\end{gathered}
$$

где $X_{(n+1)}$ - локальный функционал, $\varepsilon\left(X_{(n+1)}\right)=0$. Введем операторы

$$
W^{a}=(S, \ldots)^{a}+\int d x \varepsilon^{a b} \Phi_{A b}^{*} \frac{\delta}{\delta \bar{\Phi}_{A}} .
$$

Справедливо соотношение

$$
\frac{1}{2} \varepsilon_{a b} \omega^{b} \omega^{a} X_{(n+1)}=\left(\frac{1}{2} \varepsilon_{a b} W^{b} W^{a} X_{(n+1)}\right)_{(n+1)} .
$$

Прямой проверкой (с учетом уравнений $(35),(36))$ можно убедиться в справедливости соотношения

$$
\frac{i}{\hbar}\left(\frac{1}{2} \varepsilon_{a b} W^{b} W^{a} X_{(n+1)}\right) e^{\frac{i}{\hbar} S}=-\frac{i}{2} \varepsilon_{a b}\left[\bar{\nabla}^{b}(\hbar),\left[\bar{\nabla}^{a}(\hbar), \frac{1}{\hbar} X_{(n+1)}\right]\right] e^{\frac{i}{\hbar} S}
$$

Принимая во внимание равенство (39), мы получаем, что действие $S_{1}^{\prime}$,

$$
e^{\frac{i}{\hbar} S_{1}^{\prime}}=e^{\frac{i}{2} \varepsilon_{a b}\left[\bar{\nabla}^{b}(\hbar),\left[\bar{\nabla}^{a}(\hbar), \frac{1}{\hbar} X_{(n+1)}\right]\right]} e^{\frac{i}{\hbar} S_{1}}
$$

удовлетворяет $S p(2)$-мастер-уравнению и отличается от действия $S$ начиная с $(n+2)$-го приближения. Поскольку все решения совпадают в нулевом по $C$ и $B$ приближении, с использованием метода индукции получаем окончательно, что обшее локальное решение $\widetilde{S}$ для $S p(2)$-мастер-уравнения может быть построено из частного решения $S$ с помощью оператора калибровочного преобразования,

$$
e^{\frac{i}{\hbar} \widetilde{S}}=K(\hbar) e^{\frac{i}{\hbar} S},
$$


т.е. калибровочные преобразования действуют на решениях $S p(2)$-мастер-уравнения транзитивно, причем можно ограничиться операторами калибровочных преобразований вида $(37)$ с $\nabla=\nabla_{0}$.

Так же, как и в п. 5.2, можно проверить, что операторы $\bar{\nabla}^{a}$ и операторы калибровочных преобразований (37) являются квазиклассическими приближениями соответственно формальных нильпотентных операторов $\bar{\Delta}^{a}$ и операторов калибровочных преобразований вида $(37)$ с

$$
U=\frac{1}{2} \varepsilon_{a b}\left[\bar{\Delta}^{b},\left[\bar{\Delta}^{a}, \Delta\right]\right]
$$

Напомним, что дифференциальные выражения для канонических операторов $\bar{\Delta}^{a}$ и $\Delta$ совпадают с соответствующими выражениями для операторов $\bar{\nabla}^{a}$ и $\nabla$. При этом действие квантовой теории формально должно удовлетворять квантовому $S p(2)$-мастеруравнению

$$
\bar{\Delta}^{a}(\hbar) e^{\frac{i}{\hbar} S}=0
$$

или

$$
\frac{1}{2}(S, S)^{a}+\int d x \varepsilon^{a b} \Phi_{A b}^{*}(x) \frac{\delta}{\delta \bar{\Phi}(x)_{A}} S=i \hbar \Delta_{2}^{a} S .
$$

Дифференциальное выражение для $\Delta_{2}^{a}$ совпадает с дифференциальным выражением для $\nabla_{2}^{a}$.

Благодарности. Б. Л.В. благодарит за поддержку Российский фонд фундаментальных исследований, грант № 99-01-00-376. И.В.Т. благодарит за поддержку Российский фонд фундаментальных исследований и Комиссию европейского сообщества, гранты РФФИ № 99-01-00980, INTAS № 96-0308. Ш.С.Ш. благодарит за финансовую поддержку Российский фонд фундаментальных исследований и Комиссию европейского сообшества, гранты РФФИ № 99-02-17916, INTAS №96-0308, РФФИ-INTAS № 95-829.

\section{Список литературы}

[1] I. A. Batalin, G. A. Vilkovisky. Phys. Lett. B. 1981. V. 102. P. 27.

[2] G. Leibbrandt. Rev. Mod. Phys. 1975. V. 47. P. 847.

[3] И. А. Баталин, И. В. Тютин. ТМФ. 1998. Т. 114. С. 250.

[4] Н. Н. Боголюбов, Д. В. Ширков. Введение в теорию квантованных полей. М.: Наука, 1976.

[5] М. А. Наймарк. Теория представлений групп. М.: Наука, 1976.

[6] G. Barnich, F. Brandt, M. Henneaux. Commun. Math. Phys. 1995. V. 174. P. 57.

[7] П. М. Лавров, И. В. Тютин. Изв. вузов. Физика. 1982. № 7. С. 64; ЯФ. 1985. Т. 41. С. 1658.

[8] I. A. Batalin, P. M. Lavrov, I. V. Tyutin. J. Math. Phys. 1990. V. 31. P. 1487.

[9] И. В. Тютин, Ш. С. Шахвердиев. ТМФ. 1998. Т. 115. С. 373.

Поступила в редакцию 3.II.1999 г. 